\title{
Design of a Mathematical Model for Spectrum Utilisation in Cognitive Radio
}

\author{
B. S. Olanrewaju \\ Department of Computer Science Wellspring \\ University \\ Benin-City, Nigeria
}

\author{
O. Osunade \\ Department of Computer Science University of \\ Ibadan \\ Ibadan, Nigeria
}

\begin{abstract}
The explosive growth of wireless communication systems and computing devices is being hindered by the scarcity of readily available usable frequency band as most bands are licensed and the unlicensed bands are becoming overcrowded. Research has however, shown that a considerable portion of licensed bands lies idle at some points in time or location. This prompts the development of cognitive radios (CRs) to improve the overall utilisation of the limited spectrum by opportunistically using licensed spectrum. This paper observed that the two approaches of underlay and overlay of spectrum utilisation of CRs are not maximising the potentials of CRs. To further improve spectrum utilisation, a hybrid model of the two approaches is hereby presented. The model uses the principles of match filtering and interference temperature. The design is implemented within the scope of IEEE 802.22 research group which describes the coexistence of cognitive radios with TV bands. This paper therefore, presents a mathematical formalism that allows CRs to utilise the TV band while ensuring a minimum interference to the primary users.
\end{abstract}

\section{General Terms}

Wireless communication

\section{Keywords}

Wireless communication, dynamic spectrum management, cognitive radio, IEEE 802.22.

\section{INTRODUCTION}

Wireless technology depends on the limited radio frequency which is a part of the electromagnetic spectrum. Radio frequency spectrum is in the middle of the electromagnetic spectrum and it covers a frequency range of about $10 \mathrm{KHz}$ to $300 \mathrm{GHz}$ [1]. A large and growing part of the world's output relies upon use of spectrum for large varieties of things. According to [2], wireless technology is proliferating rapidly with over 3 billion wireless devices, most of which are cell phones and mobile computers, and the number is expected to increase to approximately 100 billion by the year 2025 .

The key purpose of spectrum management is to maximize the value that society gains from the radio spectrum by allowing as many efficient users as possible while ensuring that the interference between different users remains manageable [1]. Spectrum management provides each user with the right to transmit on a particular frequency over a particular area, typically in the form of a licence. In a licensed spectrum, only the licensee has the right to use the spectrum in that particular area. A few bands designated as unlicensed spectrum, allow users to share spectrum but with restriction to limit the consequences of interference [1,3]. Spectrum allocation is necessary in order to ensure interference free operation for each radio service. However, when exclusive right (licensed spectrum) is given to a particular user to operate in a particular frequency, some sort of inefficient usage of the spectrum is inevitable. This is because the spectrum lies idle when the licensee is not using the spectrum and this limit the introduction of new technologies by supposedly shortage of spectrum which in much of the time sits idle [1]. Researchers have however, shown that most allocated spectrum sits idle at any given time $[3,4]$. The observation is that most of the licensed spectrum at some time in a particular location lies fallow when the primary user is not transmitting while the unlicensed spectrum band is increasingly being overcrowded $[5,6]$.

The increasing demand for spectrum in recent years due to the introduction of new wireless technology has put pressure on spectrum manager to evaluate spectrum utilisation for the purpose of identifying idle channel for re-use [7, 8]

In order to improve the efficiency of spectrum, radio systems are allowed to coexist in the same spectrum through dynamic spectrum access (DSA). Effective spectrum utilisation is achieved when one device transmits while others are idle. Dynamic spectrum access sharing could be between Primary and Secondary users (i.e. licensed and unlicensed users) or among different secondary users in open spectrum band.

In order to meet the demand for spectrum, new technologies to efficiently utilize the limited resource are being developed. One of these technologies is cognitive radio which is designed to opportunistically use licensed spectrum when licensed users are inactive $[2,5]$.

The current spectrum sensing techniques in cognitive radio are not maximizing the opportunistic usage of the spectrum. In the overlay approach shown in Figure 1, CR uses the spectrum only when licensed users are not present. Even when primary users' usage of the spectrum is low, CR cannot use it at all when usage at restricted transmission would not have cause any harmful interference.

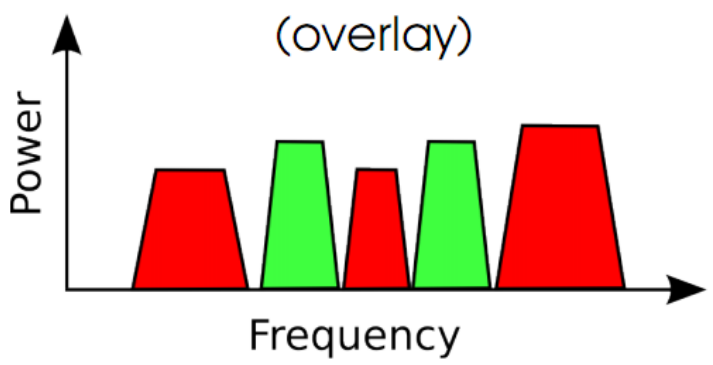

Figure 1: Overlay Sharing of Spectrum. Source: [12] 
In the underlay approach shown in Figure 2, CRs are allow to share and coexist with primary users in the spectrum but with the constraint that CRs users cannot transmit above a set limit, so as not to cause harmful interference to primary users. The shortcomings of this approach is that when primary users are not even present, CRs users still cannot transmit above the set limit even though doing so will not affect primary users as they are inactive during the period.

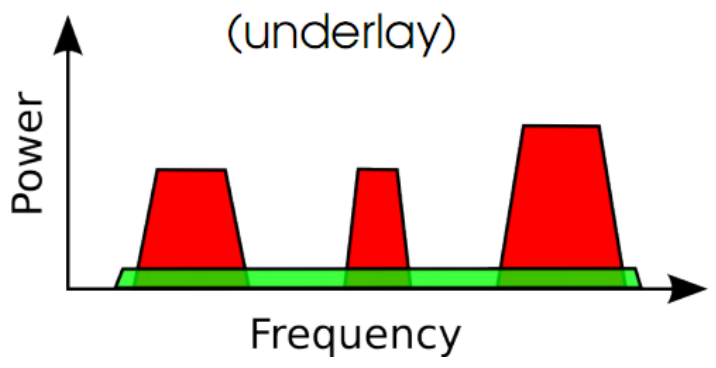

Figure 2: Underlay Sharing of Spectrum Source: [12] According to [9], because the current approach to spectrum access has not kept up with spectrum demand, there appears to be scarcity of electromagnetic spectrum. However, the introduction of cognitive radios has significantly affected spectrum management policy which has led to the idea of opportunistic spectrum utilisation for finding available spectrum to improve coverage and capacity $[9,10]$. Finding available spectrum requires the ability to sense the absence or presence of licensed users and this is a major challenge for cognitive radios [11].

Different sensing techniques classified as overlay and underlay approaches have been developed. [12] identified the shortcomings of these approaches and proposed a hybrid model where the need to opportunistically and maximally use the underutilized licensed spectrum band was justified. The model proposed addresses how to ensure that cognitive radios, through a sensing technique and interference temperature model, use vacant spectrum optimally when licence users are not transmitting. In the hybrid model, cognitive radio users will be able to use the available spectrum optimally when the licensed users' absence is noted unlike in the existing model where cognitive radio users are restricted to the set fixed transmitting parameters.

This paper now presents a mathematical model of the hybrid approach.

\section{LITERATURE REVIEW}

\subsection{Introduction}

Data communication refers to information or signal generated in one way or the other from a source and transmitted to a desired destination or receiver external to message source [13]. The distance over which data moves may vary from a few thousandths of an inch, as is the case within a single IC chip within a computer to as much as several thousands of kilometres in communications satellites $[13,14]$. Wireless communications systems are systems that use and rely on the scarce radio frequency spectrum which is the fields of electrical and magnetic energy that carry communications signal to transmit data. Wireless communications systems have been having great impact on human endeavours and activities since the time Guglielmo Marconi, in 1895 first transmitted wireless signal in the form of wireless telegraphy over a distance of three kilometres [15]. According to [16], wireless communications technology is the fastest growing segment of communications industry because of its wide range of applications in use today. Among many applications of wireless communications systems are mobile communication (voice, data and video), Internet-enabled cell phones, smart homes and appliances, remote control, interconnection of computing devices, video teleconferencing, meteorological and scientific uses, radio astronomy, Radio and Television broadcasting.

\subsection{Dynamic spectrum access}

Dynamic spectrum access is identified as an important nearterm opportunity for efficient spectrum usage and introduction of new wireless services [2]. DSA is the use of licensed spectrum opportunistically by unlicensed user when the primary user is idle or inactive and it is important in the sense that it improves the efficient utilisation of radio spectrum and allows introduction of new wireless services [2].

The challenges of DSA include developing wireless devices that can operate in different frequency bands, protecting the right of licence holders and maintaining service quality [2]. To achieve the goals of DSA, systems involves in spectrum sharing must be able to know whether to transmit or not in order not to cause interference with systems coexisting in the network. With emerging technologies like cognitive radios, opportunistic access is becoming more practical, but significant research challenges remain [17]. The $5 \mathrm{GHz}$ unlicensed frequency band is a candidate for a large set of radio services, and is one of the unlicensed frequency bands that may be efficiently used only with established spectrum etiquette [18].

The basic premise of DSA is that spectrum licence holders, known as primary users, should allow other devices, known as, secondary users to transmit in their frequency bands when they are not using them, on a non-interference basis, for example, idle TV spectrum can be reused for regional broadband access, which is the goal of IEEE 802.22 [19].

There are two basic dynamic spectrum access approaches, overlay and underlay approach in CRs. In the overlay approach, the SUs access the portion of the spectrum that is not used by PUs as a result; there is virtually no interference to the PUs. In the underlay approach, the SUs access the network by spreading their signals over a wide frequency band. The underlay approach imposes severe constraints on the transmission power of SUs. Operating below the noise floor of PUs, the SUs are allowed to interfere with PUs up to a certain tolerable level.

The overlay approach is not very effective if cognitive radio techniques' inherent need to increase the spectrum utilisation is taken into consideration. So the underlay allowing the SU to use the spectrum band while the PU is operating on the spectrum is more appropriate. In this case, avoiding the interference in cognitive radio as well as primary device is important.

\subsection{Cognitive Radios}

A cognitive radio is a software radio equipped with sensors and software that allow it to perceive the operating environment and learn from experience [7].

Cognitive radios by design are capable of sensing their environment and thereby make necessary changes in their transmission parameters to use the spectrum when primary 
users are inactive and to leave when licence users reappear [2, $9,10,20,21]$. From software defined radio (SDR) which was conceived by Joe Mitola in 1991, comes the idea of cognitive radio as an option to the problems inherent in SDR [9]. Cognitive radio is a technology for spectrum sharing that recognizes vacant bands of spectrum and adjusts autonomously its transmission to one of the bands by using SDR technology [10].

Cognitive radio technology is expected to improve spectrum access through: increased spectrum efficiency of licensed spectrum users; secondary markets by allowing licensees to lease their spectrum access e.g. by machine-controlled negotiation between systems; automated frequency coordination between licensees; opportunistic spectrum use by unlicensed devices while protecting incumbents from harmful interference [7].

Radio functionalities are major challenges in cognitive radio as it requires a considerable amount of intelligence from the transmitting device. Cognitive radio need to detect the presence of primary users in a licensed spectrum and quit the frequency band as quickly as possible if the corresponding primary radio emerges in order to avoid interference to primary users [7]. From the definition of cognitive radio, [22] highlighted two main characteristics of cognitive radio to be cognitive capabilities and re-configurability. This means that a cognitive radio will be able to detect multiple idle frequency bands and decides which frequency band to use since it is in most cases unrealistic that the user will be able to pick the right band [2]. In other word, cognitive radio systems require the four major management functions of spectrum sensing, spectrum decision, spectrum sharing and spectrum handoff or mobility [23].

Cognitive radio network is an intelligent multi user wireless communication system that knows, understand and learn the process of transmission from its environment and thereby reconfigure the transmission parameters of each transceiver in the network to facilitate and control communication processes among multiple users through cooperation and proper allocation of available resources. Cognitive radio networks intend to address the inefficient usage of spectrum in static allocation through dynamic sharing of available spectrum between licensed users also called primary users and unlicensed users. To address the challenges of dynamic spectrum access, there is need for the description of CR network architecture for the development of communication protocols that will help in the realization of the goals of cognitive radio networks [22].

The different implementations of cognitive radio network architecture as recorded by [9] includes CR1 and DARPA's $\mathrm{XG}$ architecture which are examples of ontological reasoning radios, biologically inspired cognitive radio and CORTEKs which are examples of non-deterministic procedural radios, the last cognitive radio presented by [9] is XG1, which is an example of a deterministic procedural radio.

However, [22] classified components of the CR network architecture into two groups, the primary network and the CR network. The primary network (or licensed network) is referred to as an existing network, where the primary users have a licence to operate in a certain spectrum band. Due to their priority in spectrum access, the operations of primary users should not be affected by unlicensed users. Because CR network does not have a licence to operate in a desired band, it requires additional functionality for $\mathrm{CR}$ users to share the licensed spectrum band, base stations that provide single-hop connection to $\mathrm{CR}$ users and spectrum brokers that play a role in distributing the spectrum resources among different $\mathrm{CR}$ networks [22]

\subsection{Spectrum Sensing Techniques in Cognitive Radio}

The spectrum sensing techniques in cognitive radio are classified into five main categories namely, Transmitter detection, Primary receiver detection, Cooperative Sensing, Interference Temperature Management, and Other Approaches. Match filtering technique and interference temperature are employed for this model

\subsubsection{Matched Filter $(M F)$}

This sensing technique performs optimally if transmitted primary signal is known at secondary receiver. It requires perfect knowledge of primary user signalling features and this makes implementation complexity to be very high because it needs to identify different signal types [4]. When the transmitted signal is known at receiver, matched filtering (MF) is known as the optimal method for detection of primary users since it maximizes received signal-to-noise ratio (SNR) [24].

A matched filter (MF) is a linear filter designed to maximize the output signal to noise ratio for a given input signal. Matched filter operation is equivalent to correlation in which the unknown signal is convolved with the filter whose impulse response is the mirror and time shifted version of a reference signal. MF is the most complex in terms of implementation but also, has the highest accuracy among the sensing techniques [25].

\subsection{Interference Temperature Management}

Works as an ultra-wide band (UWB) technology where the SUs are allowed to transmit simultaneously with PUs using low transmit power and are restricted by the interference temperature level [25]. The disadvantage of this approach is that CR users will not be able to transmit beyond the set interference temperature limit even with the absence of PUs in the spectrum band [4]. Using this model, cognitive radios (CRs) operating in licensed frequency bands would be capable of measuring the current interference environment, and adjusting their transmission characteristics in such a way that their transmissions avoid raising the interference temperature over a regulatory limit [26]. Interference management is one of the most important topics in cognitive radio networks since secondary user ( $\mathrm{SU}$ ) is allowed to reuse the spectrum of the primary user (PU) only if SU do not cause interference and resulting degradation in the PU transmission [27].

\section{NETWORK MODELLING}

The design of the model is based on the scope of IEEE 802.22 which describes the coexistence of cognitive radios with TV bands. According to [9], with these bands in mind, the IEEE 802.22 working group is pursuing the development of a waveform intended to provide high bandwidth access in rural areas using cognitive radio techniques. It is stated that the 802.22 standard intends to achieve spectral efficiencies of up to $3 \mathrm{bits} / \mathrm{sec} / \mathrm{Hz}$ corresponding to peak download rates at coverage edge at $1.5 \mathrm{Mbps}$. Simultaneously, the 802.22 system hopes to achieve up to $100 \mathrm{~km}$ in coverage [28]. 
According to [19], there are two main challenges in implementing the network model. The first involves identifying licensed signals and the second problem involves measuring the interference temperature $T_{I}$ in the presence of a licensed signal. These two problems are addressed in the existing models of overlay and underlay upon which this paper develops a hybrid model to increase spectrum utilisation.

\section{MATHEMATICAL MODEL}

The optimal way for any signal detection is by using a matched filter (figure 3 ) since it maximizes received signal-tonoise ratio [29]. The primary users are identified by matching the priory information about their transmission characteristics to the features extracted from the received signal. In many broadcast signals such as television, a pilot carrier is transmitted to aid in phase synchronization [30].

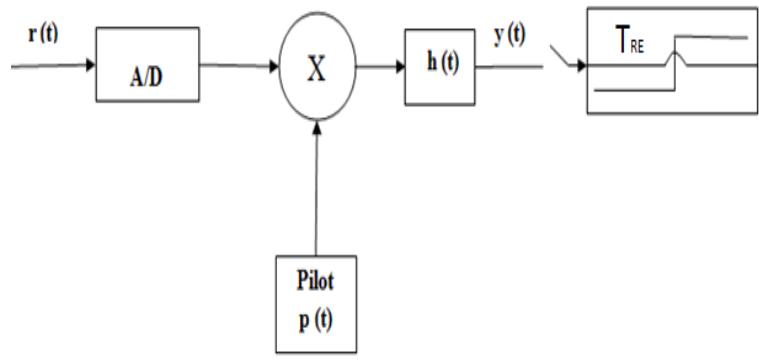

Figure 3: Matched filter Using pilot Signal for signal detection

Supposed $r(t)=s(t)+n(t)$

Where

$r(t)$ is the input to the filter

$\mathrm{s}(\mathrm{t})$ is the signal with a finite duration $\mathrm{T}$ and known waveform $p(t)$ to be detected

$\mathrm{n}(\mathrm{t})$ the additive noise

The Match filter impulse response to the signal is given as:

$\mathrm{y}(\mathrm{t})=\int_{0}^{t} r(t) h(t) d t$

$h(t)$ is the impulse response of the filter

$y(t)$ is the output of the matched filter

The matched filter is designed to maximize the ratio of the square of signal amplitude to the square of the noise amplitude. This is done by using a matched filter with optimum impulse response $h_{\text {opt }}(t)$. This is found thus:

$\mathrm{SNR}=\frac{y_{S}^{2}(t)}{y_{n}^{2}(t)}=\frac{\text { signal energy }}{\text { noise variance }}$

The signal part of the output is given in equation (4) and its expression in terms of amplitude and pulse duration is given in equation (5).

$\mathrm{y}_{\mathrm{s}}(\mathrm{t})=\int_{0}^{t} s(t) h(t) d t$

The received signal $\mathrm{s}(\mathrm{t})=\mathrm{A}_{\mathrm{p}} \mathrm{P}_{(\mathrm{t}-\mathrm{to})}$
Where $A_{p}=$ unknown signal amplitude, and $\left(t-t_{0}\right)=$ Pulse duration, $\mathrm{T}$

The Fourier transform of $\mathrm{s}(\mathrm{t})$ in equation (5) is given as

$S(f)=A p P(f) e^{-j 2 \Pi f t 0}$

The peak pulse amplitude A, of the received signal could be derived from the Inverse Fourier Transform (IFT) of the signal part as

$$
\begin{aligned}
\mathrm{A} & =F^{-1} \int_{0}^{f} H(f) S(f) d f \\
& =\mathrm{A}_{\mathrm{p}} \int_{0}^{f} H(f) P(f) e^{j 2 \pi f t} d f
\end{aligned}
$$

The noise part of the output and its Fourier Transform are given in equations (9) and (10)

$y_{\mathrm{n}}(\mathrm{t})=\int_{0}^{t} n(t) h(t) d t$
$Y_{n}(f)=\int_{0}^{f} H(f) G_{n}(f) d f$

The noise variance can be expressed as

$\sigma^{2}=\int_{0}^{f}[H(f)]^{2} G_{n}(f) d f$

Maximising the ratio of peak output amplitude A to the noise, gives:

$\left(\frac{A}{\sigma}\right)^{2}=A_{p}^{2} \frac{\left[\int_{0}^{f} H(f) P(f) e^{j 2 \pi f t}\right]^{2}}{\int_{0}^{t}[H(f)]^{2} G n(f) d f}$

$\left(\frac{A}{\sigma}\right)^{2}=A_{p}^{2} \int_{0}^{t} \frac{[P(f)]^{2}}{G_{n}(f)} d f$

Assuming that $G_{n}(f)$ is a white Gaussian noise with spectral density of $N_{0} / 2$, equation (13) reduces to

$\left(\frac{A}{\sigma}\right)_{\text {max }}^{2}=\frac{2 A_{p}^{2}}{N_{0}} \int_{0}^{f}[P(f)]^{2} \mathrm{df}=\frac{2 E_{p}}{N_{0}}$

Where $E_{p}=A_{p}^{2} \int_{0}^{f}[P(f)]^{2} \mathrm{df}$

The signal at the output of the filter, at the observing instant $t$ is given by

$y_{t}=\frac{2 E_{p}}{N_{0}}$

$y(t)$ is then compared with a threshold $T_{R E}$

\subsection{Determination of Threshold}

There are two possible ways to determine the threshold $\left(\mathrm{T}_{\mathrm{RE}}\right)$ of a signal. One way is to estimate the energy of the signal and reduce it to half, fix it as a threshold. Another way is to compute the standard deviation of the signal by computing the mean and use it as threshold. Of the two methods, the former one is theoretically proved to be optimal.

If the known primary signal energy is $\mathrm{E}_{\mathrm{s}}$, then

$\mathrm{T}_{\mathrm{RE}}=\frac{E_{S}}{2}-------(17)$

If $\mathrm{y}(\mathrm{t})>\mathrm{T}_{\mathrm{RE}}$ then primary signal is present

If $y(t)<T_{R E}$ then primary signal is absent 
The interference temperature $T_{I}$ can be specified as a function of bandwidth $\mathrm{B}$ as

$$
\begin{aligned}
& T_{I}\left(f_{c}, \mathrm{~B}\right)=\frac{1}{B K} P_{I}\left(f_{c}, \mathrm{~B}\right) \text {------- (18) } \\
& =\frac{1}{B K}\left(\frac{1}{B} \int_{f_{c-\frac{B}{2}}}^{f_{c+\frac{B}{2}}} S(f) d f\right) \text {------- (19) } \\
& =\frac{1}{B^{2} K} \int_{f_{c-\frac{B}{2}}}^{f^{c+\frac{B}{2}}} S(f) d f
\end{aligned}
$$

$P_{I}\left(f_{c}, B\right)=$ average interference power in watts at centre frequency $\mathrm{f}_{\mathrm{c}}$, and bandwidth $\mathrm{B}$

$\mathrm{K}=$ Boltzmann's constant $\left(1.38 \times 10^{-23} \mathrm{~J} / \mathrm{K}\right)$

Where $\mathrm{S}(\mathrm{f})$ represents spectral power of current $\mathrm{RF}$ environment.

Given $\mathrm{B}$, to compute a valid $\mathrm{P}$, If $\mathrm{n}$ (number of primary users) $=0$ :

$\mathrm{P} \leq \mathrm{P}_{\max }$, the radio's maximum transmit power.

For $\mathrm{n}>0, \mathrm{P}$ is given as:

$\mathrm{P} \leq\left(\frac{B_{i} K}{M}\left(T_{L}\left(f_{i}\right)-T_{I}\left(f_{i}, B_{i}\right)\right)\right)$-------- $(21)$

The goal is that the interference temperature present added to the interference to be introduced by the SU will not exceed $\mathrm{T}_{\mathrm{L}}$, that is:

$\mathrm{T}_{\mathrm{I}}\left(\mathrm{f}_{\mathrm{i}}, \mathrm{Bi}\right)+\frac{M i P s u}{K B i} \leq \mathrm{T}_{\mathrm{L}}\left(\mathrm{f}_{\mathrm{i}}\right) \quad \forall 1 \leq i \leq n$

This power requirement of the $\mathrm{SU}$ is then computed as:

$\mathrm{P}_{\mathrm{su}} \leq\left(\frac{B_{i} K}{M_{i}}\left(T_{\mathrm{L}}\left(\mathrm{f}_{\mathrm{i}}\right)-\mathrm{T}_{\mathrm{I}}\left(\mathrm{f}_{\mathrm{i}}, \mathrm{B}_{\mathrm{i}}\right)\right)\right)$

$\mathrm{P}_{\mathrm{su}}=$ Unlicensed transmitting power

$\mathrm{M}_{\mathrm{i}}=$ Fractional value between 0 and 1 representing attenuation between SU and PU (depends on distance between primary user transmitter and secondary user transmitter).

If $\frac{2 E_{p}}{N_{0}}>\mathrm{T}_{\mathrm{RE}}$ then,

$\mathrm{P}_{\mathrm{su}} \leq\left(\frac{B_{i} K}{M_{i}}\left(T_{\mathrm{L}}\left(\mathrm{f}_{\mathrm{i}}\right)-\mathrm{T}_{\mathrm{I}}\left(\mathrm{f}_{\mathrm{i}}, \mathrm{B}_{\mathrm{i}}\right)\right)\right)$ else,

$\mathrm{P}_{\mathrm{su}}=\mathrm{P}_{\max }$

Where

$\mathrm{P}_{\mathrm{su}}$ is the transmission power of the cognitive radio with the presence of primary user signal and $\mathrm{P}_{\max }$ is the maximum transmission power when the primary user is absent.

\section{CONCLUSION}

This research has developed a new spectrum utilisation technique which is the Hybrid of Overlay and Underlay methods. The Hybrid method uses match filtering principles and interference temperature management in Cognitive radios. This hybrid technique is to be implemented in the Digital Television (DTV) band. When the research is successfully completed, the contribution will be improved spectrum utilisation in the TV band where cognitive radio will not only coexist with the DTV signal in the overlay but also in the underlay in a hybrid mode. This will be possible in the sense that in the case of the presence of primary users, cognitive radio will operate within a set limit. Also, when primary users' are not using the spectrum, instead of restricting the cognitive radio to the set limit, it will be able to opportunistically use the spectrum maximally.

\section{FUTURE WORK}

This mathematical model is to be implemented by designing an algorithm that will be used by the media access control of cognitive radio.

\section{REFERENCES}

[1] Cave, M., Doyle, C., \& Webb, W. 2007. Essentials of Modern Spectrum Management. New York: Cambridge University Press.

[2] Steenkiste, P., Sicker, D., Minden, G., \& Raychaudhuri, D. 2009. Future Directions in Cognitive Radio Network Research. NSF Workshop Report. Retrieved July 11, 2011, from http://www.cs.cmu.edu/ prs/NSF_CRN_Report_Final.pd $f$

[3] Peha, J. M. 2008. Sharing Spectrum through Spectrum Policy Reform and Cognitive Radio. In Proceedings of the IEEE special issue on Cognitive Radio.

[4] Danda, B. R. \& Gongjun, Y. 2011. Spectrum Sensing Methods and Dynamic Spectrum Sharing in Cognitive Radio Networks: A Survey. International Journal of Research and Reviews in Wireless Sensor Networks. Vol. 1, No. 1.

[5] Patrick, M. 2008. Cognitive Radio: A Survey. University of Waterloo. Retrieved July 13, 2011, from http://www.cst.uwaterloo.ca/DSS/presentations_files/20 08_Patrick_Mitran.pdf.

[6] Pasi, L. \& Aleksi P. 2009. Survey on Performance Analysis of Cognitive Radio Networks. Retrieved July 13, 2011, from http://www.netlab.tkk.fi/tutkimus/abi/publ/cogsurvey2.p $d f$

[7] Kimmo, K. 2004. Spectrum Sharing and Flexible Spectrum Use. FUTURA Workshop 16.8.2004. Retrieved Aug. 10, 2011, from http://www.personal.psu.edu/bxg215/spectrum\%20sensi $n g . p d f$

[8] Australian Communications and Media Authority (ACMA). May, 2011. Towards 2020-Future spectrum requirements for mobile broadband. Retrieved July 13, 2011, from

http://www.acma.gov.au/webwr/_assets/main/lib312084 /i fc13_2011_toward_2020 future_spectrum_requirements.pdf.

[9] James, O. N. 2006. Analysis and Design of Cognitive Radio Networks and Distributed Radio Resource Management Algorithms. Diss. Electrical Engineering, Virginia Polytechnic Institute and State University.

[10] Yuguchi, K. 2008. Impact of Cognitive Radio Technology on Spectrum Management Policy. International Telecommunications Society, 17th Biennial Conference. Retrieved July 13, 2011, from http://www.canavents.com/its2008/abstracts/184.pdf

[11] Yonghong, Z.,Ying-Chang L., Anh, T. H., \& Rui, Z. 2009. A Review on Spectrum Sensing for Cognitive Radio: Challenges and Solutions. European Association for Signal Processing (EURASIP) Journal on Advances in Signal Processing, Volume 2010: Article ID 381465, 15 pages. Hindawi Publishing Corporation. doi:10.1155/2010/381465

[12] Olanrewaju, B.S. \& Osunade, O. 2012. Proposed Interference Temperature Model for Improved Spectrum 
Efficiency in Cognitive Radios. In the proceedings of EIE's $2^{\mathrm{ND}}$ International Conference on Computing, Energy, Networking, Robotics and Telecommunications, 2012. Pp72-80

[13] Strangio, C. E. 2006. Data Communications Basics- A Brief Introduction to Digital Data Transfer. CAMI Research Inc., Acton, Massachusetts. Retrieve from Retrieved July 2, 2011, from http://www.camiresearch.com/Data_Com_Basics/data_ com_tutorial.html

[14] Williams, B.K., \& Sawyer, S.C. 2003. Using Information Technology: A Practical Introduction to Computers \& Communications. New York: McGraw-Hill/Irwin.

[15] Gans, J. S., Stephen, P. K. \& Wright, J. 2005. Wireless Communications. Handbook of Telecommunications Economics, Volume 2. Retrieved Aug. 22, 2011 from http://profile.nus.edu.sg/fass/ecsjkdw/WirelessCommuni cations_Final.pdf.

[16] Goldsmith, A. 2005. Wireless Communications. Cambridge University Press. Retrieved Aug. 22, 2011, from

http://wsl.stanford.edu/ andrea/Wireless/SampleChapte rs.pdf

[17] Peha, J. M. 2007. Emerging Technology and Spectrum Policy Reform. International Telecommunications Union (ITU) Workshop on Market Mechanisms for Spectrum Management, ITU Headquarters, Geneva, January 2007.

[18] Xing, Y., Chandramouli, R., Mangold, S., \& Sai, S. N. 2006. Dynamic Spectrum Access in Open Spectrum Wireless Networks. IEEE Journal on Selected Areas in Communications, Vol.24, No.3, March 2006. pp 626637

[19] Clancy, T.C. 2007. Formalizing the Interference Temperature Model. Wiley Jjournal on Wireless Communications and Mobile Computing. Retrieved July 18, 2011, from http://onlinelibrary.wiley.com/doi/10.1002/wcm.482/ $p d f$.

[20] Clancy, T.C. 2006. Dynamic Spectrum Access in Cognitive Radio Networks. Diss. Faculty of the Graduate School of the University of Maryland . ix +106 . Retrieved July 11, 2011, from http://www.cs.umd.edu/ jkatz/THESES/clancy.pdf.

[21] Narayan B. M. 2008. Cognitive Radio Networks. Princeton ACM / IEEE-CS Chapters. January 2008 Joint
Meeting. Retrieved July 13, 2011, from http://princetonacm.acm.org/meetings/mtg0801.pdf

[22] Akyildiz, I. F., Won-Yeol, L., Vuran, M. C., \& Mohanty, S. 2008. A Survey on Spectrum Management in Cognitive Radio Networks. IEEE Communications Magazine Cognitive Radio Communications and Networks April 2008: Pp 40 - 48.

[23] Wang, L. \& Wang C. 2010. Spectrum Management Techniques with QoS Provisioning in Cognitive Radio Networks. In Proceedings of IJCSIS Journal of Computer Science September 2010

[24] Proakis, J. G. 2000. Digital Communications, 4th ed. Boston, MA: McGraw Hill.

[25] Mansi, S. \& Gajanan, B. 2011. Spectrum Sensing Techniques in Cognitive Radio Networks: A Survey. International Journal of Next-Generation Networks (IJNGN) Vol.3, No.2, June 2011

[26] Clancy, T.C. and Arbaugh W.A. 2007. Measuring Interference Temperature. Retrieved July 18, 2011, from citeseerx.ist.psu.edu/viewdoc/download?doi=10.1.1.1 45.5368 .

[27] SENDORA. 2010. Test Report on Sensing Algorithm Implementation. Retrieved Aug. 10, 2011, from http://www.sendora.eu/system/files/SENDORA_D3.2_ve rsion1.1_part1.pdf

[28] Cordeiro, C., Challapali, K. and Birru, D. 2006. IEEE 802.22: An Introduction to the First Wireless Standard based on Cognitive Radios. Journal of Communications, Vol. 1, No. 1, April 2006

[29] Cabric, D., Mishra, S.M., and Brodersen, R.M. 2004. Implementation Issues in Spectrum Sensing for Cognitive Radios. Berkeley Wireless Research Center, University of California, Berkeley. Retrieved May 17, 2012 , from http://citeseerx.ist.psu.edu/viewdoc/download?doi=10.1. 1.110.2154\&rep=rep1\&type $=$ pdf

[30] Wild, B. and Ramchandran, K. Detecting Primary Receivers for Cognitive Radio Applications. 1st IEEE International Symposium on New Frontiers in Dynamic Spectrum AccessNetworks. Baltimore, 8-11 November 2005 , pp.124-130. http://www.eecs.berkeley.edu/ dtse/3r_ben_dyspan05.pd f 